\title{
The Environment of a Hyper-Connected Network Society and Occupational Ethics
}

\author{
Su-Won Kim \\ Korea Research Institute for Vocational Education and Training, Sejong-si, South Korea
}

\begin{abstract}
The hyper-connected network society is a society environment in which all people-people, people-objects, things-things can be connected quickly and intelligently. In this environment of the hyper-connected network society, anyone can easily get the information they want, and the information and the knowledge will be a source of wealth. However, in the environment of a hyper-connected network society, the advanced information technology can harm human dignity or life if the information communication technology is used unfavorably (distortion, manipulation) by crime technique to satisfy the personal greed or pay back the grudge. Therefore, in this study, it established the direction of related occupational ethics in preparation for the hyper-connected network society in order to enable ethical values to be applied to experts and stakeholders in each field. And it made some suggestions necessary for the establishment of relevant policies at the national/social level. As a result of this study, it was found to be necessary: (1) personal information and privacy protection, (2) fair and autonomous ethical consciousness, (3) technology development for public interest, (4) enactment of ethical code for each field, (5) interests and strategic contrasts for occupational ethics of national dimension, (6) social learning (home-school-work) of occupation ethics, (7) changes for basic principles and virtue of work ethics in the hyper-connected social environment.
\end{abstract}

Keywords: hyper-connected network society, occupational ethics, job structure, job description

\section{Introduction}

The building of the advanced national network and the collection and utilization of data through this are essential to secure the national competitiveness of a future in the area of public, business, and smart (Smart X) for the intelligent information society and the 4th Industrial Revolution. The data of vast amounts collect and transmit by being connected sensors, terminals, mobile vehicles, etc., to the networks, and the networks that promote the creation of new business and the social innovation are expected to develop into "the neural networks of social systems" by connecting all people and things.

In this environment of the hyper-connected network society, anyone can easily get the information they want, and the information and the knowledge will be a source of wealth. And the tasks in almost all division will be quick, simplified, globalized, and it will be possible to receive various educational services.

However, in the environment of a hyper-connected network society, the advanced information technology can harm human dignity or life if the information communication technology is used unfavorably (distortion,

Su-Won Kim, Ph.D. in Business Administration, Korea Research Institute for Vocational Education and Training, Sejong-si, South Korea. 
manipulation) by crime technique to satisfy the personal greed or pay back the grudge. And it can be exemplified the national harm such as intelligent crime, software piracy, machine domination, etc., and the social harm such as unhealthy information distribution, computer virus spreading, hacking, etc., the personal harm such as personal information leakage, privacy violations, misjudgement due to incorrect information, etc., which caused by the unauthorized use of information technology.

In order to fundamentally prepare for these unethical problems that may arise in the environment of a hyper-connected network society, it is necessary to prepare the measures to set up and apply the occupational ethics that are considered to be more important than ever in the business world before enacting laws, regulations, and laws related with it.

The purpose of this study is to establish the direction of related work ethics in preparation for the environment of hyper-connected network society in order to enable ethical values to be applied to experts and stakeholders in each field. And it made some suggestions necessary for the establishment of relevant policies at the national/social level.

In order to achieve the purpose of this study, the research method that we conducted is as follows. First, in order to examine theories and systems related to setting the occupational ethics in the environment of a hyper-connected network society such as super-intelligence, job ethics, job consciousness, and job attitude, and other intelligence information of society's ethics guidelines, etc., the related literature and data were collected and analyzed. Second, in order to find out the issues on job structure and job content, characteristics, major subjects, types and contents of occupational ethics expected in the hyper-connected network society, the two delphi-surveys were conducted for 33 people including the officials of ICT company, the specialists (researchers, etc.) of ICT and job, the officials of relevant government departments. The two delphi-surveys distributed and collected questionnaires at a certain percentage of respondents using e-mail, facsimile, and various social networking (SNS). The opinions were grouped by items after conducting the survey of open-ended type (the primary), and the second survey was conducted as the multiple-choice form based on this.

\section{Review on the Theories and the Systems Related to the Hyper-Connected Network Society and Occupational Ethics}

\section{Significance and Characteristics of the Hyper-Connected Network Society}

The hyper-connected network society is a society environment in which all people-people, people-objects, things-things can be connected quickly and intelligently (Oh, 2017). Namely, the hyper-connected network society is that the hyper-linked network connects the objects and the space as objects and the existence of the real world in which humans live, and the hyper-connected objects and spaces become the environment surrounding an human life and integrate the cyber world and the real world. In the hyper-connected network society, it creates a value-creating network that creates, collects, shares, and utilizes the huge information in order to provide the user-centered intelligent services by connecting the objects of various physical space and the process and data contents of virtual space through the internet.

The ultimate purpose of the hyper-connected network society is that people live happily, and the happy lives are the result when people's social needs are met. People have been constantly developing their skills for a comfortable life. It is difficult to derive an integrated values or norms in a hyper-connected network society because these technologies and people's desires work together to create values and norms.

In the hyper-connected network society, we will create a new order by providing a platform in the network 
where the small-scale creative business is generated and developed explosively by the demonstration and the convergence of services and business such as smart home, smart car, smart education, smart medical, realistic media, etc., through the $\mathrm{R} \& \mathrm{D}$ and the global cooperation. On the other hand, the nature of hyper-connection is the basis for creating a new culture and value through creating the other social services different from existing ones.

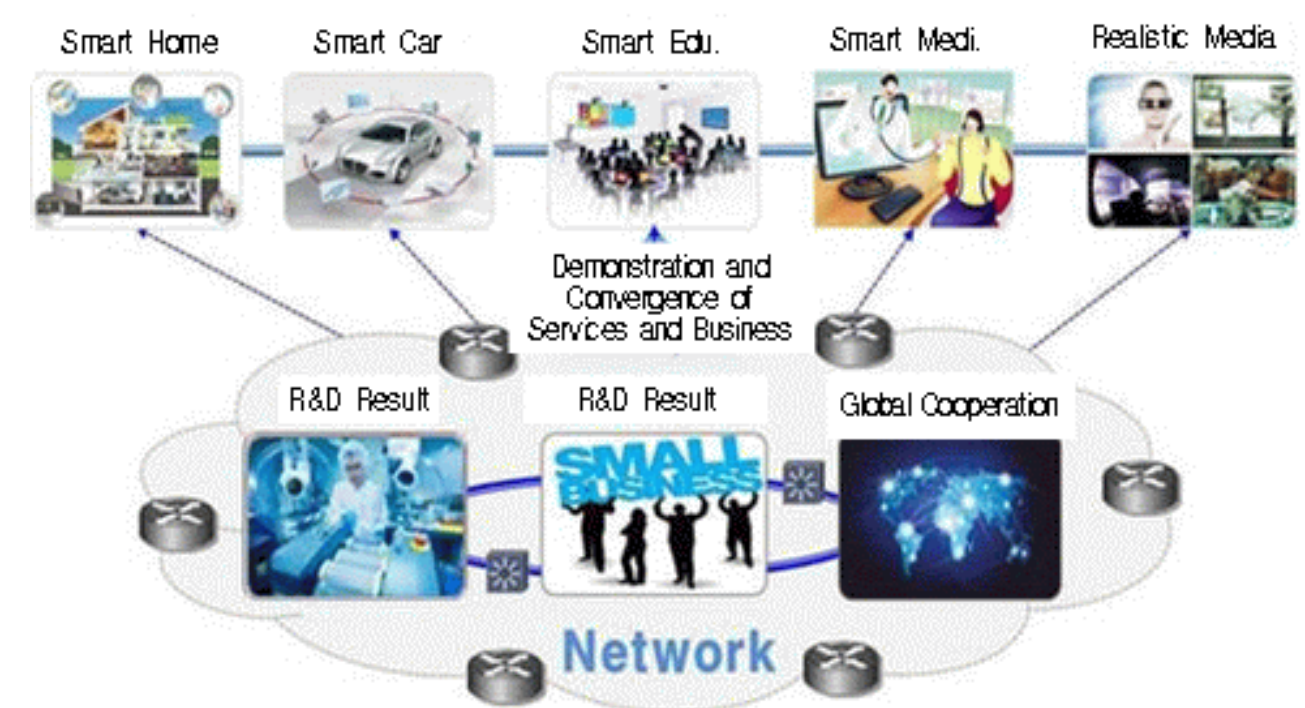

Figure 1. Roles of the hyper-connected network in a hyper-linked era. Source: The Electronic Newspaper (January 12, 2017).

In this respect, horizontal connection, open ecosystem, creation of synergy, long-term perspective, collaboration-oriented connection, and creative and voluntary individuals, etc., will gather together to create a community culture with their own way in the hyper-connected network society. In addition, it is a place to express the human desire for self-expression and fulfillment of self-fulfillment desire by using social network, and the self-expression will lead to self-realization in transparent and repeated society.

On the other hand, in the 2012 Global Information Technology Report of the World Economic Forum (WEF), the hyper-connected network society is the characteristics of always on, readily accessible, information rich, interactive, and not just about people (Lee, Kim, Kim, \& Choi, 2015).

If you look at the problems that are commonly raised in these societies, there are various problems that can not be solved legally due to artificial intelligence, big data, ICT crime, production and dissemination of false information, privacy violation, and intellectual property right. Therefore, in order to be a happy hyper-connected society, we need a new concept of occupational ethics that can discriminate between right and wrong. In particular, it is imperative to establish a sense of responsibility and accountability ethics for various behaviors conducted in anonymity.

\section{Significance and Characteristics of Occupational Ethics}

In general, occupational ethics defines to act in accordance with reason of the relationship between people and people in occupational behavior. In the hyper-connected network society, because artificial intelligence controls between people and people, a person is bound by the hyper-connected network and can act as programmed regardless of his or her will. Therefore, it is difficult to explain the concept of occupational ethics 
until now because the ethical problem newly emerging in the hyper-connected network society will be as diverse as the utilization of the intelligent hyper-connected network. In particular, various behaviors that are performed anonymously will present various problems that are difficult to solve legally.

In general, an ethics in the hyper-connected network society can be based on the teleological ethics (Doosan Encyclopedia, 2011) ${ }^{1}$ that pursues maximum happiness at minimum cost and the ethical theory of utilitarianism that aims for maximum value of happiness pursuit by nature such as always on, readily accessible, information rich, interactive, and not just about people. However, there are limitations in asserting the ethics of the hyper-connected network society by the theory of teleological ethics because the technological problems or the hyper-connected network society may cause harm to human beings depending on the personal desires of developers, suppliers, and there are limitations in claiming the theory of utilitarian ethics because the majorities who monopolize an information can ignore the minorities or violate the human rights (Oh, 2017; Kim, 2018).

If so, can Kant's deontological ethics (Sin, Park, An, Nam, \& Lee, 2012) ${ }^{2}$ explain the ethics in a hyper-connected network society? The Kant's moral law has two sides: One is to make the most reasonable action based on rational reason, that is, if we understand our duty, we must move that duty to action. The other is that we do treat them with the purpose and not treat them with means when treating people, which is applied to the ethics of developer. However, there is a possibility that the content developed in good faith may be used as malice in some cases (Oh, 2017).

In the hyper-connected network society, the data as a core resource contain an ethical dilemma that combines protection principles and utilization principles because the importance of data (a special form of intelligence and information) is privileged. Therefore, in the hyper-connected network society, the complex values such as digital citizen ethics are needed instead of one value or ethics, and the reliability with fairness and accuracy of knowledge information and the establishment of occupational ethics according to industry-specific characteristics are needed. The General Data Protection Regulation (GDPR), which was enacted in the EU Parliament in 2016 and executed in May 2018, is an empirical example showing how protection and utilization of data should be improved in the hyper-connected network society by improving previous the personal information protection laws. The GDPR is focused on the protection of personal information in terms of the structure of the law, and it is clear how operators should implement and take responsibility for privacy protection.

\section{Object and Type of Occupational Ethics}

Occupational ethics in the hyper-connected network society is a coexisting of traditional ethics, cognitive ethics, and technical ethics. Our society has changed along with the business ethics as the industry develops. Internet ethics came into being due to the deepening and expansion of the 3th Industrial Revolution and the emergence of a network society through rapid development of the internet. Here, as the 4th Industrial Revolution began, a new term "artificial intelligence ethics" emerged, and this ethics was called "ethics of the

\footnotetext{
${ }^{1}$ Teleology refers not only to human behavior, but also to the philosophical position that all events and phenomena occurring in the world are defined in purpose. The assumption is that not only human conscious behavior, but also all events and phenomena of nature in the world, are defined for purpose, and it is the way of thinking based on these assumptions. Ethical teleology is classified into various special forms such as hedonism, selfishness, utilitarianism, etc., by the content of purpose and their opponents.

2 Deontology is that it is human duty and good to act according to the law that determines right. Duty means respect for the law of morality, the inevitability to act according to the law. And so, the fundamental problem in the deontological ethics theory is what is the law of reasonable action everywhere transcending time and space.
} 
intelligence information society" or "ethics of the 4th Industrial Revolution" by naming changed society as "intelligence information society" (Kim, 2017). The today's society has become a society of remarkable change that is spread over time and space to all humans all over the world through the intelligent internet including the possibility of human transcendence such as artificial intelligence. However, with the development of science and technology, the human desire for comfort can be continuously satisfied, but it is a society that requires a new work ethics that human beings should not do harm to people in the workplace.

Occupational ethics in the hyper-connected network society can look at the scope from the position of developers, suppliers, and users in the cognitive aspects, and based on technologies corresponding to the device layer, the network layer, the platform layer, and the content layer in the technical aspects by layer (Oh, 2017; Kim, 2017).

Occupational ethics by cognitive (Kim, 2017) is based on the precautionary principle that protects social systems from the potential risks of the hyper-connected network and the intelligence information technology, and to provide a norms of ethics that can alleviate the cognitive risk factors and the side effects in terms of human welfare, authority, and expanding of freedom. It proposes specific action guidelines for areas that require ethical discipline as a concrete measure, and contributes to the formation of self-regulatory environment and the growth of industry. For sustainable development, it proposes criteria that do not place undue burden on developers and suppliers by cognitive distinction. It also contributes to participation of user and strengthening of authority, and develops to help capacity enhancement of user.

Occupational ethics by layer distinction (Oh, 2017) should be able to lead the complex society in a complex society where technologies such as hyper-connected network and societies interact closely. The layer consists of a device layer that emphasizes the deontological ethics theory and Rawls' justice theory, a network layer that consistently applies the values of efficiency based on teleological ethics theory, a platform layer that has to worry whether or not to be governed by certain norms, and a content layer that emphasizes the value of human beings based on moral ethics theory.

\section{A Case Study of Occupational Ethics in the Environment of a Hyper-Connected Network Society}

\section{Pin-Tech Industry}

The most important business principle of the pin-tech is functional decomposition (unbundling). It is a strategy to concentrate on a particular function through breaking down a number of functions of a modern bank. Figure 2 illustrates such strategies of the pin-tech startups.

Pin-tech has few real-life face-to-face relationships because it fundamentally utilizes ICT and data technologies. And, in many cases, because it is global service oriented, it is often free from the restrictions of individual countries. For this reason, besides universal occupational ethics, one should have special occupational ethics. And so, the pin-tech companies create and implement the business models based on these specific occupational ethics.

The pin-tech companies deliberately do not create separate financial accounts, and often use existing accounts such as banks and securities firms. If a separate financial account is opened, the risk of exposure to numerous illegal hacking could put consumers in serious danger. We have seen a number of recent cases to put consumers at risk that the Security Exchange to use the most advanced technology, namely Block Chains, has been targeted for hacking attacks. For similar reasons, many pin-tech firms deliberately limit the transaction 
amount. A small amount of transactions can clearly restrict the economics of illegal hacking and prevent deviation of internal employees. In addition, an ecosystem that promotes the convenience of transactions, raises the frequency of transactions, and seeks to become a living culture is fostered. These efforts are for platform formation, and the network effect creates an ecosystem in which unique occupational ethics can be systematically implemented.

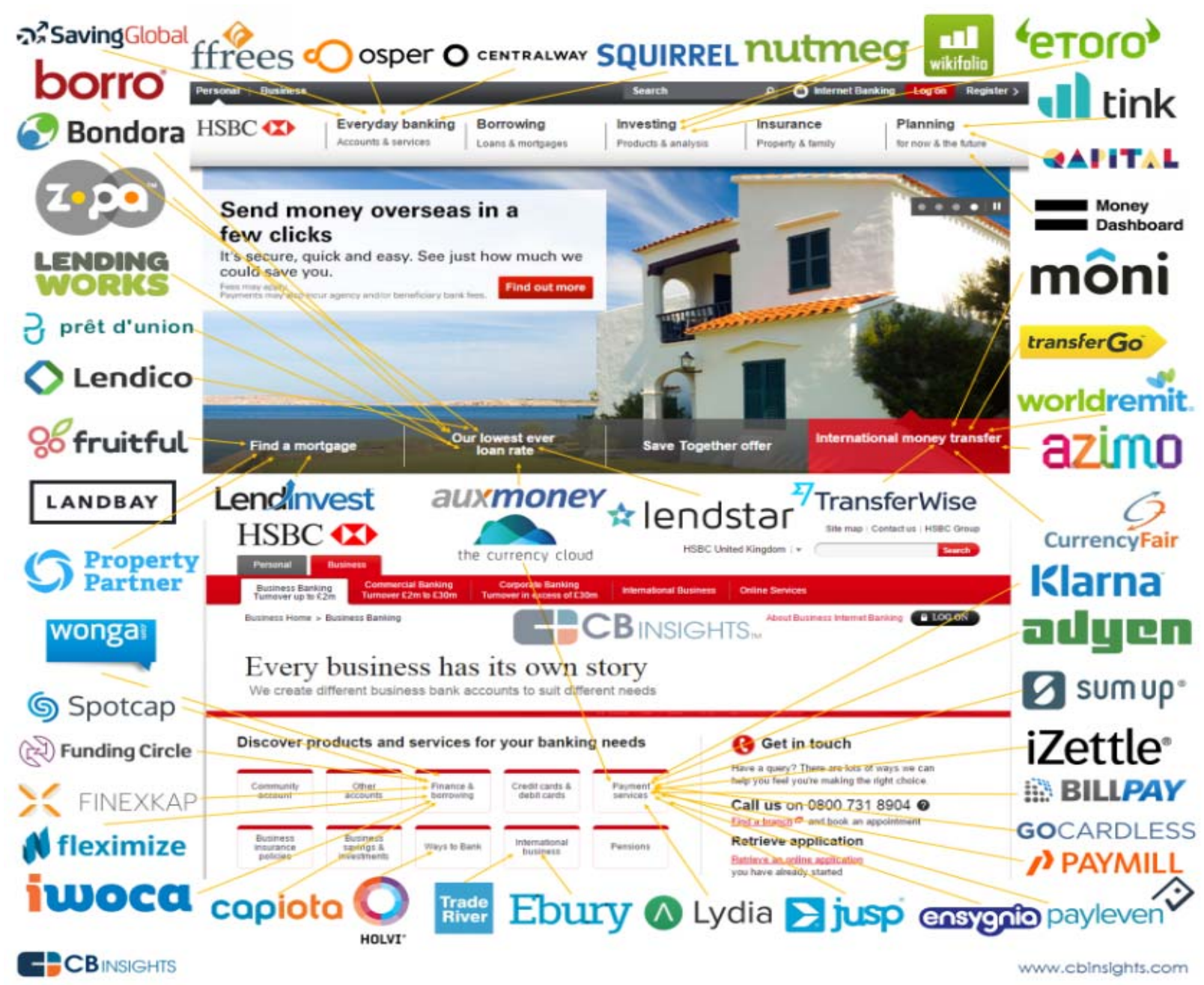

Figure 2. Unbundling of pin-tech. Source: CBInsights Homepage (2018. 11). www.cbinsights.com.

\section{Edu-Tech Industry}

Along with pin-tech in the United States and Europe, the fastest growing industry is edu-tech. Since the "Ken Robinson Report" was submitted in the UK in 1998, the " 21 st century education" has been fully discussed, and the new 4C (creativity, communication, critical thinking, and collaboration) capabilities have been agreed (Sir Ken Robbins Homepage, 2018). And the "2030 Learning Framework" was announced at the OECD in 2018; it set digital literacy, data literacy, and healthcare literacy as the direction of future education in addition to traditional literacy (literacy power, illiteracy) education (OECD Homepage, 2018).

In the United States and Europe, the edu-tech companies have established organic relationships with schools, and are providing tools for innovation and educational innovation. In pin-tech, a service model can be completed by unbundling, but edu-tech needs further functional rebundling. From the teacher's or student's 
point of view, not only can service functions be not utilized seamlessly without functional re-bundling, but the data must be inter-joined. For this reason, the edu-tech ecosystem is divided into educational cloud companies and startups offering a variety of functional services.

The U.S. education authorities selected education cloud providers after bidding by the state education office, and the Google was chosen by the largest number of states (New York Times Homepage, 2018). Educational data are still classified as sensitive information and often have limitations on utilization, but the use of GDPR case applies an increasing in the U.S.

The edu-tech companies, which focus on the role of teachers' applicants, are ethically free. Especially, it is more so when it is linked to a standard curriculum. However, edu-tech, which must be based on students' educational data or has the ability to innovate the education process, faces an ethical dilemma. It is a completely different matter between to provide analysis information to the teacher in charge and to provide analysis information to students after analyzing a particular student's education data. The teachers with relatively strong occupational ethics can try to be the main body of problem solving and implement appropriate prescription. However, because this is not the only case, we need a mechanism to prevent every one of them from failing study or in school life. The problem of subject matter and structure in occupational ethics is typical here. In this way, the work ethics of teachers and edu-tech companies, despite many similarities, exists at cross-strait branches.

The present form of existence of edu-tech is that in addition to the educational cloud, there are also maker education and school innovation (school BPR). In schools where there is a maker's education, they create maker-space as a separate space for teachers and professionals, and teachers or experts may conduct the classes or delegate the education to local communities by forming a social learning system. Here the ethical dilemmas of teachers or professionals are related to the various guarantees of opportunity and stickiness of choice in a student's career path.

In the field of lifelong education besides school education, in cases, the market provides education services using edu-tech. In the private education market area, a balanced occupational ethics are important because economic efficiency is important as well as the effectiveness of education. Unlike schools, the education market needs constantly supply innovation because there is none or lacks steady ecological support such as government budgets. In many countries, even the private education market, implementing a system such as education voucher to maintain the ecological balance is to solve the ethical dilemma of market failure.

\section{Analysis of Issue on Job Structure, Job Description, and Occupational Ethics in the Environment of Hyper-Connected Network}

In this section, there are analyzed and summarized the issues related to job structure, job description, and occupational ethics in the hyper-connected network society focusing on the results of Delphi Survey as shown in Figure 3.

\section{Issue on Job Structure and Job Description}

The core technology of the hyper-connected network society is matched with the core technology of the 4th Industrial Revolution such as Robot that combines Artificial Intelligence (AI) technology, Internet of Things (IoT), Cloud Computing, and Big Data. These technologies will be combined and fused with other technologies, and support and develop the networking of all things and people. Hereupon, the source 
technology industry that utilizes the hyper-connected network, the IoT, and the cloud-based software, and the industry that provides services by these technologies have developed, and network advancement, smart data, smart services (smart home, smart car, smart medical, etc.) will be formed.

Due to the nature of the hyper-connected network, there are formed a number of trust-based and the consultative and collaborative industrial structures based on ICT technology. As the industry changes of the existing value-chain form are accelerated, the large-scale manufacturing industry will be transformed into a cell-linked manufacturing industry. In the case of the service industry, it is expected that the service industry of the new paradigm will appear with the choice of the consumer rather than the choice of the service provider. In addition to simple information analysis by artificial intelligence and big data, the machines equipped with artificial intelligence will be done on behalf of people from the dangerous work and the data processing of large amounts to the labor and the services requiring complex judgment.
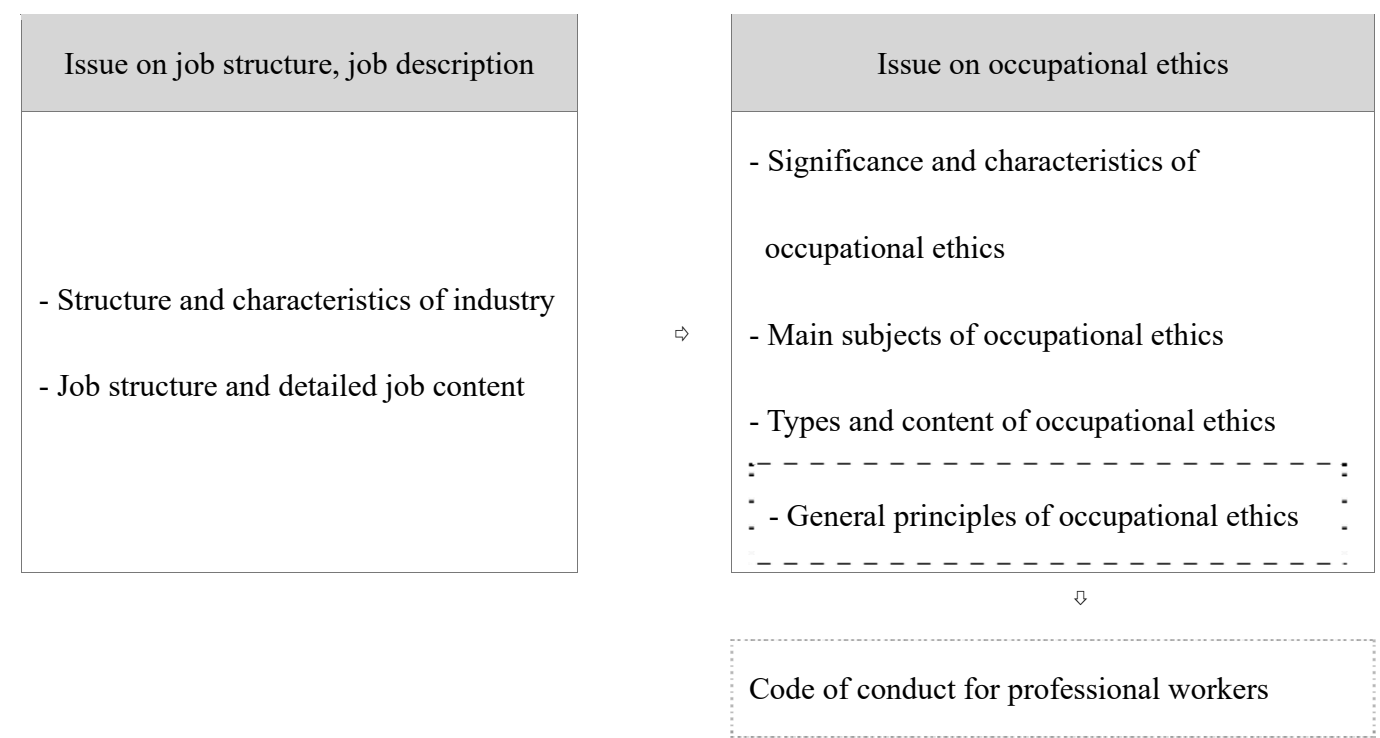

Figure 3. Linkage between the issues related to job structure, job description, and occupational ethics.

With the basic knowledge of artificial intelligence, big data, and software technology, etc., new duties are merged with the existing duties. And as the technology of artificial intelligence and the technology in other fields are combined and combined, it will be created the new jobs related to this and the various duties related to life-style that emphasizes the quality of life. Basically, a duty to build a hardware infrastructure, and a duty to operate these infrastructures, a duty to collect, analyze, and process the produced data, and a duty to analyze the advanced data to predict and utilize based on big data, etc., will be developed. With expansion of the service through data analysis based on big data, it will be changed to a duty on cloud-based sharing rather than the hierarchical transmission method. A duty to process the linked-data, a duty to develop and utilize the technology related linked-equipment and network, and a duty to plan and market the linked-data will be formed. In addition, the job category related the creative occupations or the ICT such as data processing and robot coordinators to support the learning of artificial intelligence will be increased, and the jobs related to simple/repetitive office administration or low-skilled work will be decreased.

In the hyper-connected network society, most of the jobs are small and the life-cycle of the jobs is short, but they will be specialized with a very high level of knowledge and skills. As a result, the job structure will be 
transformed into a form in which a group of expert experts in each area is composed rather than a vertical form, and performs the work through collaboration. Furthermore, we will move away from the horizontal and vertical job structure and work in a grid-like structure, that is, work while moving organically according to the duties required in the work centering on the content and the quality of knowledge and experience. In addition, the work style will change to a variety of personal-focused forms of work such as the flex time, the tele-commuting, and the cloud services through internet.

As for details of the job description, the duties to which the hyper-connected technology is applied will be different depending on whether they belong to collection, processing, storage, utilization, disposal, or security of information. The duties related to collecting will include the job contents related to sensor communication and wireless communication. The duties related to processing and storage will need the job experience and the knowledge such as cloud and big data. The duties related to utilization will need the competency of information technology such as platform development or artificial intelligence. In addition, the hardware technology for safety disposal and safety security of information, and the know-how technology of information protection will be needed. The field of programming (coding) and software manufacture such as artificial intelligence, internet of things, robotics, cloud computing, big data, and block chain, etc., will be developed, and the duties related to new technologies such as augmented reality (AR), virtual reality (VR), 3D printing, robotics, biotechnology, and nanotechnology, etc., will be developed. Many parts are expected to be automated unlike the maintenance and the repair by manpower in the existing industry, but the maintenance of core technologies and algorithms (analytical engines, etc.) will need the people.

\section{Issue on Occupational Ethics}

In the context of the above, the occupational ethics that should be applied in the environment of a hyper-connected network society is as follows. First, the occupational ethics in the environment of a hyper-connected network society means the purlin of the mutual relationship that must be maintained among the social members in the continuous economic activity in order to keep human life alive, and it means norms for internal and external actions to be socially expected. For example, it is important to establish fairness and morality in the collection and utilization of information that is very common in the hyper-connected network society. Second, it is very important that the values of ICT-based science and technology and the values of human dignity are recognized without being mutually invaded. In other words, because the value of human dignity and the value of science and technology are double-edged knife, it is important for stakeholders such as engineers, etc., to have the right values and philosophy. Namely, in the hyper-connected network society, we appreciate the science and technology that enhances the quality of life, and we need the ethics that does not infringe the private life or the privacy when all the subjects are collecting, analyzing, and utilizing an information. Third, in the hyper-connected network society, it protects the information and the privacy of the individual because anyone can access people and objects through the network, and it needs community awareness and public accountability guarding and protecting so that the data and the platform can be designed, produced, managed, operated. In other words, there is a high possibility to occure the ethical problems in the hyper-connected network society, such as management of cloud data, learning of artificial intelligence, etc., besides the relationship between people and people, the relationship between people and objects. Fourth, in the hyper-connected network society, occupation ethics changes according to culture and infrastructure, but it is necessary to establish the ethical values based on humanity, and to establish fair and autonomous, the ethical 
consciousness, the responsibility, and the morality under a transparent decision-making. Fifth, in the hyper-connected network society, because the cyber world and the physical world are connected, the malicious behavior such as hacking and information leakage has a strong impact on the society as a whole. And from the perspective of various job performance and flexible human relationships, universal ethics, everyday life and cyber ethics, professional skills, and knowledge creation, it is necessary to establish the ethical awareness and the regulation because it is difficult to catch up with the environment of hyper-connected network society.

The main subject of occupational ethics to be applied in the scope of the above-mentioned occupational ethics can be set as follows. First of all, it is "engineer" who collects and analyzes large amounts of data in the process of collecting and analyzing a data, and "experts, professional worker" who have a great influence on society as a whole and the ethical and moral will to negative use of expertise. In addition, it is "data analysts, data managers" who should be careful about information security as a worker who creates new information by utilizing a data, and "supplier" who needs to offer and operate the management service by non-identifying and managing a personal information and ensuring compliance with security standards. In addition, in order to create a social atmosphere for occupational ethics and to promote it in a deliberate manner, it is "national operators" who actively seek administrative support with quick judgment and attention and who create the appropriate regulations related to disclosure of individual and national data, "law enactor" of politicians, etc., who formulate the legislation for legal safety network such as personal privacy protection, etc., and "public servants, educators" who prioritize the public profit rather than the private profit according to public identity and have to execute and comply in the field with laws enacted.

The types of occupational ethics that should be applied in the environment of hyper-connected network society can think "prohibition of hacking, virus", "prohibition of privacy infringement", "information protection", "security of computer system", "management of information and data". In the environment of hyper-connected network society, it is possible to illegally invade a computer system to retrieve stored files, to change the information at will, or to damage a computer operating system. In addition, it is very important to protect information (prevention of privacy infringement, prevention of hacking, etc.) because simple information can be processed as important information through AI, big data, etc., and false information can be distributed in public. It is also necessary to prevent the infringement of personal information and privacy, but it is also important to prevent the monopolization of information in order to increase the utilization of useful information. To this end, it is necessary to construct a security system (data encryption, transmission encryption, network security, etc.) so that the information provided by customers can be safely distributed and managed when providing IT services. In addition, it needs the main agent and the person in charge responsible for management and protection of data/information produced through robot or AI. Furthermore, the accuracy, the reliability, and the fairness of information must be secured because many people produce and utilize an information.

By reviewing comprehensively the above contents, the contents of the general principles of occupational ethics to be applied in the environment of hyper-connected network society can be set as shown in Table 1 . 
Table 1

General Principles of Occupational Ethics in the Environment of a Hyper-Connected Network Society (Example)

1. Recognize and respect not only ourselves but also others' intellectual property rights, privacy, and diversity in cyber-space and reality-space.

2. Do not use personal information and copyrighted content without permission. In addition, when using other people's information, the purpose of use shall be notified and it shall be discarded immediately if the purpose has been achieved.

3. Should not be monopolized by a particular agency or individual because general information is a public good.

4. Do not write or distribute content that is harmful to mankind and society.

5. Respect the life of a member of society, and do not encroach on their privacy.

6. Avoid the acts that may infringe on personal life or information.

7. Pursue veracity, non-biasing, completeness, and fair representation of the information that he/she provides in cyber-space.

8. The public nature of the collection, provision, and utilization of information shall be secured, and the responsibility for protection of information, prevention of monopoly, truthfulness, and fairness shall be fulfilled.

9. In view of the above, in order to implement the definition of society, we must abide by rules and laws, and keep order, and faithfully fulfill our responsibilities and obligations including ethical responsibilities.

\section{Conclusion: Response Direction and Policy Suggestion of Occupational Ethics in the Hyper-Connected Network Society}

Until now, the state has not paid much attention to occupational ethics because of its excellence education, and even experts engaged in science and technology had passive ethics. However, in the environment of hyper-connected network society, there is a possibility that more serious risks may occur than technological developments in past, and there is a limit to deliberately slow down or reverse the speed of technological development in order to solve them. Therefore, I would like to set the direction of responding to occupational ethics in preparation for the environment of hyper-connected network society, and make some suggestions necessary for the establishment of relevant policies at the national/social dimension.

\section{Response Direction of Occupational Ethics}

Direction (1): In the hyper-connected network society, the artificial intelligence is developed, the various information is generated, and it can be used without permission, so the human dignity should be guaranteed. Therefore, as a specialist in the related field with the latest and the most advanced expertise in the new technology-based environment, professional workers must fulfill their responsibilities and duties with integrity and honor in their work.

Direction (2): Because it is difficult to solve ethical problems with one value in the hyper-connected network society, it should prepare specific guidelines, occupational ethics and codes of ethics suitable for professionals by job category, by layer, and by specialty. At this time, the professional workers need the ability to interact with other professionals and non-professional workers.

Direction (3): It should be analyzed the potential risk factors, and should be conducted and provided the comprehensive and exhaustive evaluation about the social impact. Therefore, the professional workers should acquire new qualities that can project their skills and abilities of critical thinking into the context of social innovation as members of civil society.

Direction (4): In the hyper-connected network society, it needs to be discussed the community-centered ethical standards for public safety and welfare in the utilization and management of information. Therefore, it should be set up a common forum participating in government, business operators, academia, and civic groups, etc., in order to establish a plan of occupational ethics.

Direction (5): An ethical value should be internalized in all aspects, including relevant technologies and 
standards, international norms, and domestic laws. However, legal norms and regulations should play a role in supplementing ethics, and should be noted for the multi-layered social impact of technological structures.

\section{Policy Suggestions}

Proposition (1): In the environment of a hyper-connected network society, the protection of personal information is essential because most information and data can be easily collected, transformed, and distributed, whether desired or not. Not only legal regulations are necessary to protect personal information, but also ethics is required to establish fairness and morality in the collection and utilization of information, and to avoid infringing other people's privacy. In addition, it is necessary to establish a security system (such as data encryption, transmission encryption, network security) so that information can be safely distributed and managed when providing IT services. On the other hand, efforts to meet international standards should be needed when a much stricter international standards in respect of personal information and privacy protection is established. In this case, it is also required to change attitudes of being equally related with the media such as artificial intelligence, which constitute the higher-connected network society that is not human-centered.

Proposition (2): In the hyper-connected network society, because of horizontal and collaborative connections, open ecosystems, creative and voluntary individualism, and accelerated technological change, etc., there are needed attention and efforts to establish a fair and autonomous ethical awareness, responsibility, and morality in terms of various job performance and flexible human networks, daily and cyber ethics, professional and knowledge creation.

To this end, we have to develop continuously the democratic political institutions and the democratic social form, and we will ensure the accuracy, the reliability, and the fairness, etc., about production and utilization of information. In addition, we should be improved that the labor contracts include the contents of occupational ethics, etc. Also, if you use the internet, you will meet many different people, but try to contact similar types of people. In this case, the people who are similar in type may feel the same opinion, echoing only the same echo. Therefore, we recognize that there are other opinions and that they can exist, and we need the education and training to have a listening to admit and a respectful attitude.

Proposition (3): In order to enhance the utilization of general information, specific institutions or individuals should not monopolize or distribute content that is harmful to mankind and society, and the technology should be developed and utilized for public good. To this end, we must secure not a single value or ethics but complex values, such as digital citizen ethics, and further we must secure the community sense and the public accountability on occupation, and a credibility with the fairness and the accuracy of knowledge information.

In addition, it is necessary to establish "The Establishment of Systematic Vocational Attitudes" that judges and depends on a social system to determine the level of confidence in the performance of duties by artificial intelligence or related experts. For example, in order for everything to be social network, the technology exists in a specific group and in a specific place, and as long as the benefits are strong, the side effects that expose unintended information are also significant. Accordingly, it is necessary to plan and utilize so as to benefit many urbanites and even many people around the city.

Proposition (4): It should be formulated the code of conduct ethics required to professionals by specialty, and be induced to practice this. In the hyper-connected network society, it consists of a group of experts by fine detail area rather than vertical form, and most duties are expected to specialize at a significant level although 
the life cycle is short. Therefore, the professional workers should observe the basic principles of honesty and sincerity, prohibition of harm, and realization of justice, etc., with an evolved job consciousness and commitment.

Proposition (5): In the hyper-connected network society, it requires attention and lead not only at the professionals of engineers, the data managers, the enterprises, etc., but also at the national level like the United States and Japan. In other words, it requires interest and participation of "the national operator" who makes appropriate regulations in relation to active the disclosure of personal and national data, and the administrative and financial support, "the lawmakers" who enact laws for the legal safety-network including the protection of privacy of individuals, and "the public servants" and "the educators" who should take priority in public interests and have to execute and comply by laws in the field according to their public status. Professional ethics is very important not only for professionals in professional work, but also for nonprofessional politicians and administrators who support and manage them.

In addition, It is necessary to prepare for the future change in working environment at the national level, and to establish the directions and the strategies of desirable institutionalization through social consensus among labor, management, government, and civic groups. Therefore, the government will enact and announce the code and the acts of professional ethics related to the hyper-connected network society. And it should be established and institutionalized a system of executive bodies (governance) to manage and support directly including enactment and compliance for the code of professional ethics. The executive body should make the institutional outlets where a variety of stakeholders can participate and reflect these opinions.

Proposition (6): It should be included doing one's best for a role that he/she was given since childhood in the curriculum, and have to develop the sensibility. Hereupon, it has to be systematized occupational ethics through the process of social learning (home, school, work, etc.) on occupational ethics. And it has to verify the social learning outcomes in homes, schools, and workplaces, and have to conduct the consulting to guide them to comply with the standards of occupational ethics.

However, in the absence of home education since the 1980s, the home has become a space for entrance examination rather than a space for education. The transition of these fundamental problems is needed. Hereupon, it is necessary to cultivate the experts for education and training and consulting on occupational ethics, and to build a national online system that can form a consensus and suggest improvements on occupational ethics from time to time. And it is necessary to verify and standardize the content and the degree for the social impact of science and technology and the degree and the extent of compliance on occupational ethics through participation of various social groups.

Proposition (7): It is desirable to change the basic principles and virtues of occupational ethics which are emphasized at present to a appropriate form in the hyper-connected network society. It is desirable to change into a direction that I am enjoying, benefiting from, and developing in the existing society rather than sacrificing. In addition, it requires a provocative ability to create something as a kind of creativity, but to innovate from the existing norms and to resist the existing authority. Also, it is necessary to think about what to future values, sensitivity to future values, and the future values of animals such as social weakness not just the humans. Also, it is honest and feels the happiness that I exist about the relationship between oneself and others, and it needs a sense of balance that is not biased toward either side and a sense of solidarity to listen to others' opinions and to recognize others. It is desirable to specify the object or go to a solidarity consciousness because the object of service consciousness is also unclear. In addition, citizenship is based on citizens' nationality in 
civic consciousness, but it needs a sense of citizenship as citizens of the world that matches all beings beyond their nationality in the future.

Meanwhile, the concept of vocation does not fit with the changes of the present age in a society to change rapidly, and the contribution is far from the purpose and also accompanied by carrying out work by doing something for the value of the group rather than the individual. It is desirable to clarify by the sense of responsibility for society and the sense of responsibility for the future, etc.

\section{References}

CBInsights Homepage. (2018. 11). Retrieved from https://www.cbinsights.com

Do, S.-Y. (2018). The 4th Industrial Revolution Era, new technology and ethics of science and technology: Future ethics models and manpower training schemes for science and technology field. The 14th Annual Science and Technology Innovation Policy Forum, Korea Federation of Science and Technology Societies, Korean Internet Ethics Association.

Doosan Encyclopedia. (2011. 12). Phenomenological dictionary. Seoul: DoosanDong-A Co.

Electronic Newspaper Homepage. (2017. 1). Retrieved from http://www.etnews.com/

Kim, M.-J. (2017). Ethics guideline of intelligent information society for the 4th Industrial Revolution Era. A Discussion Forum to Present Human-Centered Ethical Guidance During the 4th Industrial Revolution Era. Ministry of Science and ICT National Information Society Agency, Information Culture Forum.

Kim, M.-J. (2018). Business ethics guidelines using artificial intelligence. 2019 ICT Industry Outlook Conference.

Kim, S.-W., \& Jeong, T.-W. (2018). A study on occupational ethics for future network and data utilization in the environment of a hyper-connected network society. Issue Paper, 2018-03, Korea Research Institute of Vocational Education and Training.

Lee, H.-Y., Kim, H., Kim, S.-H., \& Choi, H.-S. (2015). Socio-cultural perspectives for the sustainability of hyper-connected society and the response of Korean society. Korea Information Society Development Institute.

New York Times Homepage. (2018. 11). Retrieved from https://www.nytimes.com

OECD Homepage. (2018. 11). Retrieved from http://www.oecd.org/education

Oh, T.-W. (2017). Future norms of the hyper-connected society. Digital Research, 1(1), 7-21. Korean Internet Ethics Association.

Sin, H.-G., Park, Y.-C., An, S.-R., Nam, Z.-S., \& Lee, S.-Y. (2012. 11). Police dictionary. Seoul: Bubmunsa Co.

Sir Ken Robbins Homepage. (2018. 11). Retrieved from http://sirkenrobinson.com

The White House. (2016a). Precision medicine initiative: Data security policy principles and framework. Retrieved from https://goo.gl/dH7Cvh

The White House. (2016b). White House launches public workshops on AI issues. Retrieved from https://obamawhitehouse.archives.gov/blog/2016/05/03/ 